\title{
THE LATTICE DYNAMICS OF Ni-24\%Fe ALLOY BASED ON AN EMPIRICAL MANY-BODY POTENTIAL
}

\author{
İ. AKGÜN AND G. UĞUR \\ Gazi University, Faculty of Arts and Sciences, 06500 Ankara, Turkey
}

(Received February 16, 1999; revised version May 18, 1999)

\begin{abstract}
We have developed an empirical many-body potential and used it to investigate the dynamical behaviours of fcc $\mathrm{Ni}-24 \% \mathrm{Fe}$ alloy. The new many-body potential contains both two- and three-body atomic interactions. The two-body potential is expressed by the modified form of the generalized Morse potential and the three-body potential is developed based on the new two-body model potential. The parameters defining the many-body potential for $\mathrm{Ni}$ and $\mathrm{Fe}$ were computed following a procedure similar to the method defined by Akgün and Ugur for fcc structure at the lattice constant of the alloy. The radial, tangential and three-body force constants of the alloy were calculated by the concentration averages of the computed force constants of the component metals. Finally, the phonon frequencies of the alloy along the principal symmetry directions were computed using the calculated two- and three-body force constants. The theoretical results were found to be in good agreement with the corresponding experimental values.
\end{abstract}

PACS numbers: 63.20.-e

\section{Introduction}

For more than thirty years there has been interest in the development of empirical and semi-empirical interatomic potentials for investigating the elastic, lattice, and electronic properties of metals and alloys. The works in this field have also sustained the continued interest. Furthermore, the contributory role of the many-body forces in determining the above properties of solids has been emphasized by several workers [1-5]. While many different estimates of the three-body forces [6-10] have been proposed, the two-body forces have usually been described in terms of the derivatives of an unknown interionic potential. To include the most significant contribution to the calculation of the above properties which arises from the interaction among the ion and electrons, we have developed a new empirical many-body potential (MBP) in the present work. The total interaction among the 
constituents is clivided into two parts: ion-ion and electron-ion-electron contributions. The first contribution was described by the modified form of the generalized Morse potential [11] and the second one was expressed in terms of the first contribution. The functional form and the parametrization procedure of the proposed MBP are explained in Sec. 2.

Recently the phonon frequencies for $\mathrm{Ni}-24 \% \mathrm{Fe}$ binary type-II alloy have been measured using inelastic neutron scattering at room temperature [13]. The interesting feature of Ni-Fe binary type-II alloys is that the two constituents of the alloy are in different phases, as $\mathrm{Ni}$ is in $\mathrm{fcc}$ phase and $\mathrm{Fe}$ in bcc phase at room temperature. However, the $\mathrm{Ni}-\mathrm{Fe}$ alloys form a random solution having a fcc structure [13]. Due to their structural complexity, not much theoretical work has been done on Ni-Fe alloys, to reveal the lattice clynamics. Therefore, the aim of the present work is to investigate the suitability of applying both the parametrization procedure similar to the method defined by Akgün and Ugur [12] and the proposed $\mathrm{MBP}$, to the problem of studying lattice dynamics of fcc $\mathrm{Ni}-24 \% \mathrm{Fe}$ binary type-II alloy.

\section{Theory and computation}

The total interaction energy of a system of $N$ atoms, in general, may be expressed as a many-body expansion,

$$
\phi=\phi_{2}+\phi_{3}+\ldots+\dot{\phi}_{n}+\ldots
$$

where $\phi_{2}, \phi_{3}$, and $\phi_{n}$ represent the total two-body, three-body, and $n$-body interaction energies, respectively. In this paper we have re-expressed the total interaction energy of a system simply by separating $C^{\prime}$ as

$$
\phi=\phi_{2}+C \phi_{3}
$$

where $C$ is a three-body potential parameter to be determined. In the present work the new MBP developed by Akgün and Ugur contains both two- and three-body potentials.

\subsection{Two-body model potential}

For the interatomic interactions between two atoms of a lattice the two-body model potential has been described by the modified form of the generalized Morse potential [11], and the average total two-body interaction energy per atom may be written as

$$
\phi_{2}\left(r_{i j}\right)=\frac{D}{2(m-1)} \sum_{i \neq j}\left(\frac{r_{0}}{r_{i j}}\right)^{n}\left[\beta^{m} \exp \left(-m \alpha r_{i j}\right)-m \beta \exp \left(-\alpha r_{i j}\right)\right],
$$

where $m$ and $\alpha$ control the width and the depth of the potential, respectively. $D$ is the dissociation energy of the pair of ions, $r_{0}$ is the separation of the atoms for minimum potential, and $\beta=\exp \left(\alpha r_{0}\right)$. In Eq. (3) $\left(r_{0} / r_{i j}\right)^{n}$ modifies the generalized Morse potential [11] to exhibit the correct nature of the forces, particularly at short distances. $r_{i j}$ is the interatomic distance between atoms $i$ and $j$, and $r_{i j}=a\left(m_{i j}^{2}+n_{i j}^{2}+l_{i j}^{2}\right)^{1 / 2}$, where $m_{i j}, n_{i j}, l_{i j}$ are integers representing the difference between the coordinates of $i$ - and $j$-th atoms of the lattice and $a$ is the lattice constant. 


\subsection{Three-body model potential}

In the present paper we proposed a three-body potential in terms of the new two-body model potential (3). The three-body general potential coupling the atom $i$-th with its neighbours $j$ - and $k$-th may be written as

$$
\begin{aligned}
& \phi_{3}\left(r_{i j} r_{i k}\right)=\frac{C D}{2(m-1)} \sum_{j \neq k} \sum_{i}\left(\frac{r_{0}}{r_{i j}+r_{i k}}\right)^{n} \\
& \quad \times\left\{\beta^{m} \exp \left[-m \alpha\left(r_{i j}+r_{i k}\right)\right]-m \beta \exp \left[-\alpha\left(r_{i j}+r_{i k}\right)\right]\right\},
\end{aligned}
$$

where $r_{i j}$ and $r_{i k}$ are the respective separations of the atoms $j$ - and $k$-th from the atom $i$-th. $C$ is the three-body potential parameter to be evaluated. The summations in the present calculations extend up to 10-th neighbours.

\section{Q.3. Calculation of the MBP parameters}

The parameters $\left(\alpha, \gamma_{0}, D, C\right)$ defining the MBP for Ni and Fe may be computed for fcc structure at the lattice constant of binary type-II alloy $\mathrm{Ni}-24 \% \mathrm{Fe}$, following a procedure similar to the method defined by Akgün and Uğur [12] at the equilibrium semi-lattice constant of the alloy $\left(a_{0}\right)$ :

$$
\begin{aligned}
& \left.\phi_{2}\left(r_{i j}\right)\right|_{r_{i j}=a_{0}}=\epsilon_{0},\left.\quad \phi_{2}\left(r_{i j}\right)\right|_{r_{i j}=a_{0}}+\left.\phi_{3}\left(r_{i j} r_{i k}\right)\right|_{r_{i j}=r_{i k}=a_{0}}=\phi \\
& \left.\frac{\partial \phi_{2}\left(r_{i j}\right)}{\partial r_{i j}}\right|_{r_{i j}=a_{0}}=0,\left.\frac{\partial^{2} \phi_{2}\left(r_{i j}\right)}{\partial r_{i j}^{2}}\right|_{r_{i j}=a_{0}}+\left.\frac{\partial^{2} \phi_{3}\left(r_{i j} r_{i k}\right)}{\partial r_{i j} \partial r_{i k}}\right|_{r_{i j}=r_{i k}=a_{0}}=9 c a_{0} B,
\end{aligned}
$$

where $\epsilon_{0}$ is the ionic part of the total cohesive energy $\phi, B$ is the total bulk modulus, and $c$ is a geometrical constant depending on the type of the crystal (for fcc crystal $c=2$ ). For $\mathrm{Ni}$ and $\mathrm{Fe}$ the input data used in Eqs. (5) are given in Table I.

\section{TABLE I}

Input data. $[14,15]$ for $\mathrm{Ni}$ and $\mathrm{Fe}, a$ is the lattice constant of alloy [13].

\begin{tabular}{c|c|c|c|c}
\hline \hline Element & $a\left[10^{-10} \mathrm{~m}\right]$ & $-\epsilon_{0}[\mathrm{eV}]$ & $B\left[10^{11} \mathrm{~N} \mathrm{~m}^{-2}\right]$ & $-\phi[\mathrm{eV}]$ \\
\hline $\mathrm{Ni}$ & 3.553 & 2.07 & 1.860 & 4.44 \\
$\mathrm{Fe}$ & 3.553 & 0.90 & 1.683 & 4.28
\end{tabular}

The MBP parameters $\left(\alpha, r_{0}, D, C\right)$ can be evaluated for many different values of the exponent $m$ and $n$. In order to determine the best values of the $m$ and $n$ defining the MBP for $\mathrm{Ni}$ and $\mathrm{Fe}$ we computed then the second-order elastic constants $\left(C_{11}, C_{12}, C_{44}\right)$ for fcc structure at the lattice constant of the alloy. The elastic constants can be evaluated from the well-known expressions for cubic crystals $[16,17]$ :

$$
C_{11}=\frac{a_{0}^{2}}{V_{\mathrm{c}}} \sum_{j \neq k} \sum_{i}\left[m_{i j}^{4} \frac{\partial^{2} \phi_{2}\left(r_{i j}\right)}{\left(\partial r_{i j}^{2}\right)^{2}}+m_{i j}^{2} m_{i k}^{2} \frac{\partial^{2} \phi_{3}\left(r_{i j} r_{i k}\right)}{\partial r_{i j}^{2} \partial r_{i k}^{2}}\right],
$$




$$
\begin{aligned}
& C_{12}=\frac{a_{0}^{2}}{V_{c}} \sum_{j \neq k} \sum_{i}\left[m_{i j}^{2} n_{i j}^{2} \frac{\partial^{2} \phi_{2}\left(r_{i j}\right)}{\left(\partial r_{i j}^{2}\right)^{2}}+m_{i j} m_{i k} n_{i j} n_{i k} \frac{\partial^{2} \phi_{3}\left(r_{i j} r_{i k}\right)}{\partial r_{i j}^{2} \partial r_{i k}^{2}}\right], \\
& C_{44}=\frac{1}{3}\left(2 C_{11}-C_{12}\right),
\end{aligned}
$$

where $V_{\mathrm{c}}$ is the atomic volume, $r_{i j}=a\left(m_{i j}^{2}+n_{i j}^{2}+l_{i j}^{2}\right)^{1 / 2}$, and $r_{i k}=$ $a\left(m_{i k}^{2}+n_{i k}^{2}+l_{i k}^{2}\right)^{1 / 2}$. For $C_{44}$, the relation developed by Milstein and Rasky [18] is used. Comparing the computed values with the experimental values of the second-order elastic constants we have determined the best values of the exponent $m$ and $n$ given in Table $\mathrm{II}$ for $\mathrm{Ni}$ and Fe, separately. For the determined values of $m$ and $n$ the computed parameters $\left(\alpha, r_{0}, D, C\right)$ of the MBP are given in Table III.

TABLE II

Computed elastic constants (in units $1.0^{11} \mathrm{~N} \mathrm{~m}^{-2}$ ) for $\mathrm{Ni}$ and $\mathrm{Fe}$ at room temperature.

\begin{tabular}{c|c|c|c|c|c|c|c|c|c|c}
\hline \hline \multicolumn{3}{c|}{$\mathrm{Ni}$} & \multicolumn{5}{c|}{$\mathrm{Fe}$} & \multirow{2}{*}{ Ref. } \\
\hline$n$ & $m$ & $C_{11}$ & $C_{12}$ & $C_{44}$ & $n$ & $m$ & $C_{11}$ & $C_{12}$ & $C_{44}$ & \\
\hline 0.46 & 1.74 & 2.47 & 1.51 & 1.14 & 0.95 & 3.77 & 2.30 & 1.25 & 1.11 & Pres. work \\
& & 2.47 & 1.53 & 1.22 & & & 2.30 & 1.35 & 1.17 & exp. [19]
\end{tabular}

TABLE III

Computed MBP parameters for $\mathrm{Ni}$ and Fe at the lattice constants of the alloy.

\begin{tabular}{c|c|c|c|c|c|c}
\hline \hline Element & $n$ & $m$ & $D[\mathrm{eV}]$ & $\alpha\left[10^{10} \mathrm{~m}^{-1}\right]$ & $r_{0}\left[10^{-10} \mathrm{~m}\right]$ & $C$ \\
\hline $\mathrm{Ni}$ & 0.46 & 1.74 & 0.2928495 & 2.2119 & 2.5777803 & 0.1377902 \\
$\mathrm{Fe}$ & 0.95 & 3.77 & 0.1319408 & 2.9227 & 2.5598326 & 0.4859541
\end{tabular}

\subsection{Phonon dispersion relations}

The usual secular determinant to determine the frequency of vibration of a solid is given by

$$
\left|D-M w^{2} I\right|=0
$$

where $D$ is a $(3 \times 3)$ dynamical matrix, $M$ is the ionic mass density, and $I$ is the unit matrix. In the present work the elements of the dynamical matrix $D_{\alpha \beta}$ are composed of two-body $D_{\alpha \beta}^{i}$ (pair central) and three-body $D_{\alpha \beta}^{m}$ (many-body) contributions

$$
D_{\alpha \beta}=D_{\alpha \beta}^{i}+D_{\alpha \beta}^{m} .
$$

In the case of the two-body central pairwise, the interactions are assumed to be effective up to 10 th nearest neighbours and $D_{\alpha \beta}^{i}$ are evaluated by the scheme of 
Shyam et al. [20]. The typical diagonal and off-diagonal matrix elements of $D_{\alpha \beta}^{i}$ can be found in Ref. [20]. In the case of the central interaction, the first and second derivatives of the new two-body model potential (3) provide two independent force constants, i.e. the tangential force constant $\beta_{i}$ and radial force constant $\alpha_{i}$, for the $i$-th set of neighbours

$$
\beta_{i}=\frac{1}{r_{i j}} \frac{\partial \phi_{2}\left(r_{i j}\right)}{\partial r_{i j}}, \quad \alpha_{i}=\frac{\partial^{2} \phi_{2}\left(r_{i j}\right)}{\partial r_{i j}^{2}}, \quad i=1-10 .
$$

For $\mathrm{Ni}$ and $\mathrm{Fe}, \beta_{i}$ and $\alpha_{i}$ were computed for fcc structure at the lattice constant of the alloy. For the alloy, the $\beta_{i}$ and $\alpha_{i}$ were calculated by using the linear relations

$$
\begin{aligned}
& \beta_{i}(\mathrm{NiFe})=(1-x) \beta_{i}(\mathrm{Ni})+x \beta_{i}(\mathrm{Fe}), \\
& \alpha_{i}(\mathrm{NiFe})=(1-x) \alpha_{i}(\mathrm{Ni})+x \alpha_{i}(\mathrm{Fe}),
\end{aligned}
$$

where $x$ is the concentration of $\mathrm{Fe}$ in the alloy, $x=0.24$. For $\mathrm{Ni}, \mathrm{Fe}$ and $\mathrm{Ni}-24 \% \mathrm{Fe}$ alloy the computed force constants are given in Table IV. The average mass used

TABLE IV

The computed radial $\left(\alpha_{i}\right)$ and tangential $\left(\beta_{i}\right)$ force constants.

\begin{tabular}{c|c|c|c|c|c|c}
\hline \hline Serial & \multicolumn{3}{|c|}{$\alpha_{i}\left[10^{-3} \mathrm{~N} \mathrm{~m}^{-1}\right]$} & \multicolumn{3}{c}{$\beta_{i}\left[10^{-3} \mathrm{~N} \mathrm{~m}^{-1}\right]$} \\
\cline { 2 - 7 } No. & $\mathrm{Ni}$ & $\mathrm{Fe}$ & $\mathrm{Ni}-24 \% \mathrm{Fe}$ & $\mathrm{Ni}$ & $\mathrm{Fe}$ & $\mathrm{Ni}-24 \% \mathrm{Fe}$ \\
\hline 1 & 29907.5 & 23223.6 & 28303.3 & -472.632 & -194.294 & -405.831 \\
2 & -2020.77 & -748.568 & -1715.44 & 290.967 & 95.7671 & 244.119 \\
3 & -420.277 & -128.468 & -350.243 & 43.2073 & 13.6420 & 36.1117 \\
4 & -93.4374 & -29.8128 & -78.1675 & 8.15039 & 2.78612 & 6.86296 \\
5 & -24.1530 & -8.39876 & -20.3710 & 1.87831 & 0.70982 & 1.59787 \\
6 & -7.07547 & -2.70568 & -6.02672 & 0.50265 & 0.21045 & 0.43252 \\
7 & -2.28885 & -0.96296 & -1.97064 & 0.15078 & 0.06978 & 0.13134 \\
8 & -0.80185 & -0.37041 & -0.69831 & 0.04949 & 0.02523 & 0.04367 \\
9 & -0.29907 & -0.15171 & -0.26434 & 0.01748 & 0.00978 & 0.01563 \\
10 & -0.11848 & -0.06545 & -0.10575 & 0.00656 & 0.00401 & 0.00595
\end{tabular}

in the calculations for the alloy is obtained from the relation

$$
M(\mathrm{NiFe})=(1-x) M(\mathrm{Ni})+x M(\mathrm{Fe}) .
$$

In order to determine the contribution of the three-body forces to the diagonal and off-diagonal matrix elements of $D_{\alpha \beta}^{m}$, we follow the scheme of Mishra et al. [9], where a three-body potential is used to deduce the force-constant matrix, involving a. single parameter

$$
\begin{aligned}
& D_{\alpha \alpha}^{m}=4 \gamma\left[4-2 C_{2 i}-C_{i}\left(C_{j}+C_{k}\right)\right], \\
& D_{\alpha \beta}^{m}=4 \gamma\left[C_{i}\left(C_{j}+C_{k}\right)-2\right],
\end{aligned}
$$

where $\gamma$ is the second derivative of the three-body potential $\phi_{3}\left(r_{i j} r_{i k}\right), C_{i}=$ $\cos \left(\pi a k_{i}\right)$ and $C_{2 i}=\cos \left(2 \pi a k_{i}\right)$. To compute the three-body force constant $\gamma$ of 
$\mathrm{Ni}$ and $\mathrm{Fe}$ at the lattice constant of the alloy, we limit the short-range three-body forces in the fcc system only up to first-nearest neighbours. For the alloy $\mathrm{Ni}-24 \% \mathrm{Fe}$, $\gamma$ is obtained from the linear relation

$$
\gamma(\mathrm{NiFe})=(1-x) \gamma(\mathrm{Ni})+x \gamma(\mathrm{Fe}) .
$$

The computed values of the three-body force constants $\gamma=1795.80 \times 10^{-3} \mathrm{~N} \mathrm{~m}^{-1}$ for Ni, $\gamma=11642.6 \times 10^{-3} \mathrm{~N} \mathrm{~m}^{-1}$ for $\mathrm{Fe}$, and $\gamma=4159.032 \times 10^{-3} \mathrm{~N} \mathrm{~m}^{-1}$ for $\mathrm{Ni}-24 \% \mathrm{Fe}$.

Now one can construct the dynamical matrix $D_{\alpha \beta}$ by using Eq. (8) and then solve the secular equation (7) to compute the phonon frequencies along the principal symmetry directions [100], [110]. and [111] of the alloy.

\section{Results and discussions}

In the present work, the interaction system of fcc Ni-24\%Fe alloy was considered to be composed of the two-body and three-body contributions. Therefore, we developed a new MBP and first used it, as an application, to investigate the dynamical behaviours of the binary type-II alloy, where the end members have different structures. The parameters $\left(\alpha, r_{0}, D, C\right)$ defining the MBP for pure $\mathrm{Ni}$ and $\mathrm{Fe}$ were computed following a procedure similar to the method defined by Akgün and Uğur: [12], for fcc structure at the equilibrium lattice constant of the alloy. In the mean-crystal model the equilibrium pair energy, bulk modulus, and total cohesive energy were used as the input data. Then we computed the ab initio radial $\left(\alpha_{i}\right)$, tangential $\left(\beta_{i}\right)$ and three-body $(\gamma)$ force constants for $\mathrm{Ni}, \mathrm{Fe}$ and

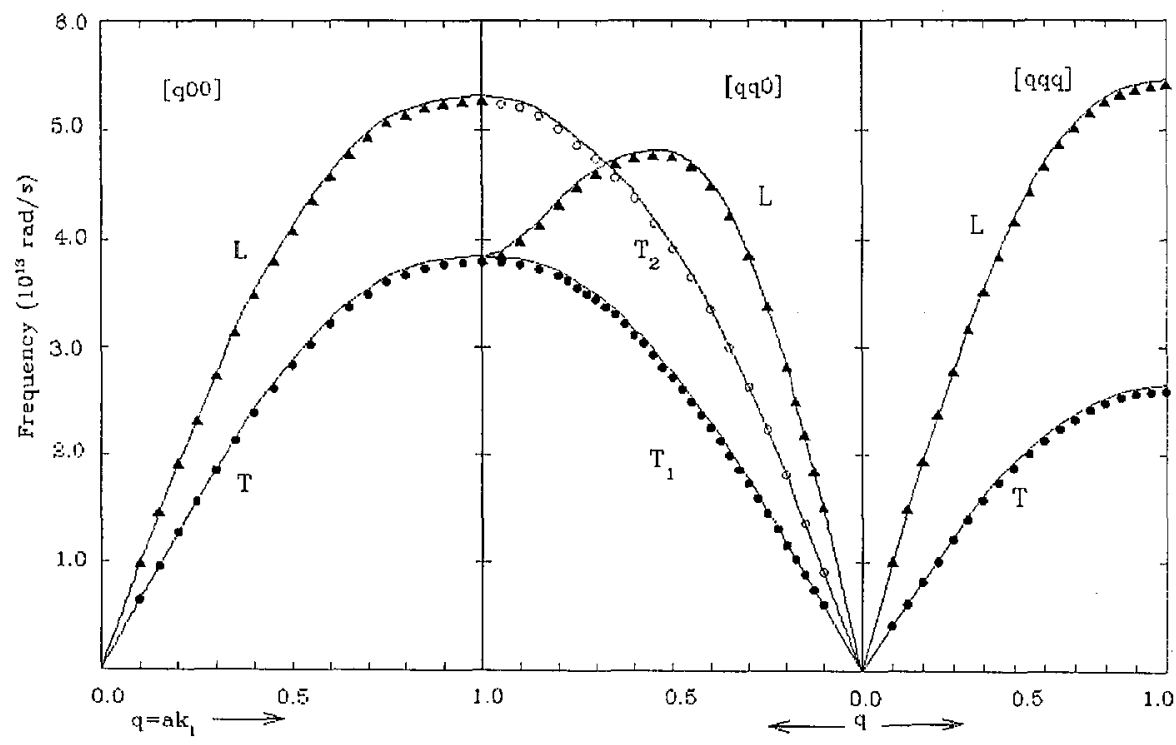

Fig. 1. Phonon dispersion curves at room temperature for $\mathrm{Ni}-24 \% \mathrm{Fe}$. The symbols $(\bullet$, full $\Delta, 0)$ represent the experimental values [13]. The solid curves show the computed dispersion curves according to the many-body interactions. 
$\mathrm{Ni}-24 \% \mathrm{Fe}$ alloy, using the new MBP. The computed values of the force constants were fed into the dynamical matrix (8) and the phonon frequencies for the alloy were calculated by solving the secular determinant (7). The computed dispersion curves are shown by solid curves in Fig. 1. Furthermore, the experimental values measured by Maliszewski and Bednarski [13] of the alloy are also shown by the symbols $(\bullet$, full $\Delta, 0)$ in Fig. 1 for comparison.

The close scrutiny of Fig. 1 suggests that the new MBP and present model reproduce all the qualitative features as well as the quantitative features of the experimental findings [13]. Furthermore, the parametrization procedure proposed in this work is credited with the following striking and meritorious features:

1. In contrast to the large number of input data used in such studies, the mean-crystal model requires only three input data, for evaluating the necessary parameters.

2. The input data being independent of the phonon frequencies and elastic constantis enhance enormously the reliability and credibility of the model and reduce the relative standard error [10] to a minimum.

3. The properly defined MBP under report has considered the capacity of evaluatimg the forces constants for any number of neighbours without needing extra inpul data. Such studies reported so far, however, require more input data for extending the interactions among further neighbours.

Consequently, the present results show that both the developed MBP and the proposed crystal model are sufficient to study the latice dynamics of the fcc binary type-II alloys.

\section{Acknowledgment}

We are grateful to the Gazi University AFP (05/98-07) for the financial support.

\section{References}

[1] J.W. Lynn, H.G. Smith, R.M. Nicklow, Phys. Rev. B 8, 3493 (1973).

[2] R. Bauer, W. Maysenholder, A. Seegar, Phys. Lett. A 90, 55 (1982).

[3] W. Maysenholder, R. Bauer, A. Seegar, Phys. Lett. A 109, 398 (1985).

[4] R.E. Beissner, Phys. Rev. B 13, 5131 (1976).

[5] D. Prakash, J.C. Upadhyaya, J. Phys. Chem. Solids 49, 91 (1988).

[6] M.S. Daw, M.I. Baskes, Phys. Rev. B 29, 6443 (1984).

[7] K.I. Chopra, D. Kitous, Solid State Commun. 79, 289 (1991).

[8] K. Mohammed, M.M. Shukla, F. Milstein, J.L. Merz, Phys. Rev. B 29, 3117 (1984).

[9] M.K. Mıshra, P. Srivastava, S.K. Mishra, Phys. Status Solidi B 171, K5 (1992).

[10] S.K. Sarkar, S.K. Das, D. Roy, S. Sengupta, Phys. Status Solidi B 83, 615 (1977).

[1.1] F. Milstein, J. Appl. Phys. 44, 3825 (197.3).

[12] İ. Akgün, G. Uğur, Phys. Rev. B 51, 3458 (1995).

[13] E. Maliszewski, S. Bednarski, Phys. Status Solidi B 200, 435 (1997). 
[14] M.D. Morse, Chem. Rev. 86, 1049 (1986).

[15] C. Kittel, Introduction to Solid State Physics, 3rd ed., Wiley, New York 1966.

[16] K. Arachana, R.P.S. Rathore, Phys. Status Solidi B 156, 77 (1989).

[17] G. Singh, R.P.S. Rathore, Indian J. Appl. Phys. 24, 303 (1986).

[18] F. Milstein, D.J. Rasky, Solid State Commun. 55, 729 (1985).

[19] R.F.S. Hearmon, in: Elastic, Piezoelectric, Pyroelectric, Piezooptic, Electrooptic Constants and Nonlinear Dielectric Susceptibilities of Crystals, Ed. K.H. Hellwege, Landolt-Bornstein, New Series, Vol. 11, Springer-Verlag, Berlin 1979, p. 11.

[20] R. Shyam, S.C. Upadhyaya, J.C. Upadhyaya, Phys. Status Solidi !' 161, 565 (1990). 